\title{
28 Research Square \\ Dual-Fano resonances and sensing properties in the crossed ring-shaped metasurface
}

Zhihui He ( $\nabla$ kazh0722@126.com )

Yan'an University

Chunjiang Li

Yan'an University

Wei Cui

Yan'an University

Weiwei Xue

Yan'an University

Zhenxiong Li

Yan'an University

Lihui Pu

Yan'an University

Jiaojiao Feng

Yan'an University

Xintao Xiao

Yan'an University

Xuyang Wang

Yan'an University

Yajie Liu

Yan'an University

Qirui Zou

Yan'an University

Yufei Yao

Yan'an University

Yixuan Niu

Yan'an University

Mengyuan Wang

Yan'an University

\section{Nano Express}

Keywords: Fano resonance, Plasmonics, Sensors 
Posted Date: February 26th, 2020

DOl: https://doi.org/10.21203/rs.2.24487/v1

License: (c) (i) This work is licensed under a Creative Commons Attribution 4.0 International License. Read Full License 


\section{Abstract}

We study dual-Fano resonances and its sensing properties in a crossed ring-shaped metasurface by use of the finite-different time-domain (FDTD) simulation. The results show that the dual-Fano resonances in the proposed crossed ring-shaped metasurface are caused by the interaction among three local surface plasmon resonances (LSPRs), and the spectra of dual-Fano resonances can be tuned by the radius of the circular ring (CR) nanostructure, the distance between the center of the two CRs in $\mathrm{x}$ direction, and the polarization of the incident light. Interestingly, single Fano resonance splits into dual-Fano resonances in the case of asymmetric ring structure arrangement or non-y-axis polarized incident or the distance $\mathrm{d}<120$ $\mathrm{nm}$. Moreover, we can also find that the refractive sensitivity in the proposed crossed ring-shaped metasurface can reach up to $1010 \mathrm{~nm} / \mathrm{RIU}$ and $1300 \mathrm{~nm} / \mathrm{RIU}$ at Fano resonance peak 1 and Fano resonance peak 2 , respectively. These results may play an important role for designing high sensitive plasmonic sensors.

\section{Introduction}

Local surface plasmon resonance (LSPR) is the collective oscillation of electrons excited by the incident light, which is confined at the surface of the metal nanoparticle [1-3]. The interaction among the different LSPRs on the adjacent nanoparticles can form the new normal modes on the surface of the nanoparticles, so many interesting phenomenon are observed based on the coupling between plasmonic resonance modes, such as plasmon induced transparency [4-10], Fano resonance [13-16], extraordinary optical transmission [17, 18], breaking the optical diffraction limit [19, 20], and so on. These peculiar optical phenomenon are widely used in surface-enhanced spectroscopies [21, 22], biochemical sensors $[23,24]$, plasmonic switching $[25,26]$, plasmonic filter [27, 28], plasmonic absorber [29-35], and so on.

Fano resonance has the asymmetrical spectra caused by the interaction between the continuous mode and discrete mode [36, 37], the sharp spectra of Fano resonance makes the strong dispersion appearing at the wavelength of Fano resonance. Thus, Fano resonance is widely used in enhancing sensing, light filed enhancement, optical storage, and so on [38-40]. And Fano resonance is studied in a variety of structure, such as metallic waveguides [41, 42], dielectric waveguides [43, 44], optical gratings $[45,46]$, graphene systems [47-48], and so on. There are also many kinds of metasurface, which can also achieve Fano resonance, such as single nanodisk, dimer and multiparticle oligomers. For the single nanodisk nanostructure, Fano resonance is often caused by introducing arranged defects, such as narrow split gap, cut-out wedge, and so on $[49,50]$. For dimer and multiparticle oligomers structures, Fano resonance is always caused by the interaction among the closely adjacent metallic nanoparticles in different size or shape [51, 52]. Nordlander et al. discussed the typical Fano resonance in plasmonic metamaterials [53]. Singh et al. investigated high-Q Fano resonance with the high sensitive sensors in metamaterials [54]. Li et al. discussed conventional plasmonic sensors and slow-light sensors based on Fano resonance in the resonator coupled waveguide [55]. Tang et al. studied Fano resonance with high sensitivity sensing in graphene coupled systems [56]. Liu et al. reported Fano resonance and its sensing performance in the pure dielectric metasurface [57]. However, there are few works about Fano resonance and its sensing 
properties in the crossed nanostructure metasurface. Different from single nanodisk, separated dimer and multiparticle, the crossed metasurface can form more possible plasmonic resonance modes, so multiple Fano resonances can be achieve in the simple crossed nanostructure metasurface.

In this paper, we proposed a crossed ring-shaped metasurface, and dual-Fano resonances and high sensitivity sensing are also discussed in the proposed metasurface through FDTD simulation method. We find that the dual-Fano resonances can be effectively tuned by the radius of the CR nanostructure, the distance between the center of the two CRs in $\mathrm{x}$ and $\mathrm{y}$ directions, and the polarization of the incident light. Especially, single Fano resonance splits into dual-Fano resonances through adjusting structural parameters and polarization directions. What is more, the maximum of sensitivity Max[s] $=1010 \mathrm{~nm} / \mathrm{RIU}$ and Max[s] $=1300 \mathrm{~nm} / \mathrm{RIU}$ for Fano resonance peak 1 and Fano resonance peak 2 are observed in the proposed crossed ring-shaped metasurface. These research results may play important roles for designing ultra-high sensitive sensors.

\section{Structure Model And Simulation Method}

We propose a crossed ring-shaped metasurface as shown in Fig. 1. The metal is chosen to be Au, and the substrate is $\mathrm{SiO}_{2}$, the permittivity of $\mathrm{Au}$ and $\mathrm{SiO}_{2}$ reference to the reported articles [58-60]. Figure 1(a) shows the schematic diagram of the proposed crossed ring-shaped metasurface, and Fig. 1(b) shows the $x-y$ plane view of the proposed metasurface. $P x=P y=400 \mathrm{~nm}$ are the length of each cell in $x$ and $y$ directions, respectively. $r_{1 \text { in }}=75 \mathrm{~nm}$ and $r_{1 \text { out }}=100 \mathrm{~nm}$ are the inside radius and outside radius for the left $R C$ structure, respectively. $r_{2 \text { in }}$ and $r_{\text {2out }}$ are the inside radius and outside radius for the right RC structure, respectively. $d$ is the distance between the center of the proposed two RCs in $x$ direction. The transmission spectra of the proposed crossed ring-shaped metasurface is simulated by use of FDTD simulation method. In our simulation process, the effective area is divided into uniform Yee cells with $\triangle \mathrm{x}=\Delta \mathrm{y}=\triangle \mathrm{z}=1 \mathrm{~nm}$ and the $\Delta \mathrm{t}=\Delta \mathrm{x} / 2 \mathrm{c}$, where $\mathrm{c}$ is the velocity of light in vacuum [61, 62], and the perfectly matched layer (PML) is chosen in this numerical simulation process [63-65]. The Gaussian beam incidents from the positive direction of $\mathrm{z}$ axis in our simulation.

Figure 1. (a) Schematic diagram of the crossed ring-shaped metasurface, (b) $x$-y plane view of the proposed crossed ring-shaped metasurface.

\section{Results And Discussions}

Firstly, we investigate the transmission responses of the proposed crossed ring-shaped metasurface when the polarization of the incident light is $x$ direction as depicted in Fig. 2 (a). We can see that a transmission dip appears at the wavelength of $994.2 \mathrm{~nm}$ (red line) when there is only left CR nanostructure arranged on the substrate. The transmission dip is caused by LSPR on the surface of left $\mathrm{CR}$. The other transmission dip appears at the wavelength of $1023 \mathrm{~nm}$ (black line), which is caused by LSPR on the right CR. Interestingly, double Fano resonances can be observed (blue line) when the left and right CRs are arranged on the substrate as shown in Fig. 2(a). Different from the separated dimer and 
multiparticle metasurface, the crossed metasurface can form more possible plasmonic resonance modes, so multiple Fano resonances can be achieve in this simple crossed metasurface. Then we study the electric field distribution Ez as shown in Fig. 2(b)-2(e). Figure 2(b) shows the electric field distribution Ez at the wavelength of $994.2 \mathrm{~nm}$ when there is only right CR configurated on the substrate, we can see that the strongly confined electric field at the right CR. The same as Fig. 2(b), Fig. 2(c) shows confined electric field at the left CR. Thus, the obvious transmission dips can be observed in Fig. 2(a). Then we describe the electric field distribution Ez at the wavelength of Fano resonance peaks. We can see that the opposite electric field distribution are shown in Fig. 2(d) and 2(e). The additional LSPR appears at the middle of structure when the two CRs are crossed with each other, so the left Fano resonance is caused by the interaction between the additional LSPR at the middle structure and LSPR at the right CR. However, the right Fano resonance is caused by the interference between additional LSPR at the middle structure and LSPR at the left CR.

Figure 2. (a)Transmission spectra of the proposed crossed metasurface, the red line shows the transmission spectra when there is only left $C R$ with $r_{1 \text { in }}=75 \mathrm{~nm}$ and $r_{1 \text { out }}=100 \mathrm{~nm}$, the black line shows the transmission spectra when there is only right $C R$ with $r_{2 \text { in }}=65 \mathrm{~nm}$ and $r_{20 u t}=90 \mathrm{~nm}$, and the blue line shows the transmission spectra when there are right and left CRs arranged on the substrate with $r_{1 \text { in }}=$ $75 \mathrm{~nm}, \mathrm{r}_{1 \text { out }}=100 \mathrm{~nm}, \mathrm{r}_{2 \text { in }}=65 \mathrm{~nm}, \mathrm{r}_{2 \text { out }}=90 \mathrm{~nm}$ and $\mathrm{d}=60 \mathrm{~nm}$. (b) The electric field distribution Ez at the wavelength of $994.2 \mathrm{~nm}$ in the case of only right CR. (c) The electric field distribution Ez at the wavelength of $1023 \mathrm{~nm}$ in the case of only left CR. (d) and (e) The electric field distribution Ez at the wavelength of $891.1 \mathrm{~nm}$ and $1004 \mathrm{~nm}$ in the case of double CRs.

Then we study the dual-Fano resonances as a function of the radius $r_{2 \text { out }}$ for the right CR with $\triangle r_{2}=r_{2 \text { out }}{ }^{-}$ $r_{\text {2in }}=25 \mathrm{~nm}$ when the polarization of the incident light is the $x$ direction as depicted in Fig. 3 (a). We can see that Fano resonance shows red shift as the radius $r_{2 \text { out }}$ increases from $80 \mathrm{~nm}$ to $120 \mathrm{~nm}$. But there is a special case when $r_{2 \text { out }}=100 \mathrm{~nm}$, it is because that the symmetric ring-shaped structure when $r_{\text {2out }}=$ $100 \mathrm{~nm}$, so the dual-Fano resonances turn into single Fano resonance. Then we discuss the electric field distribution Ez at $827.4 \mathrm{~nm}$ for $r_{2 \text { out }}=80 \mathrm{~nm}, 955 \mathrm{~nm}$ for $r_{2 \text { out }}=90 \mathrm{~nm}, 1023 \mathrm{~nm}$ for $r_{2 \text { out }}=110 \mathrm{~nm}$, and $1045 \mathrm{~nm}$ for $r_{2 \text { out }}=120 \mathrm{~nm}$ as shown in Fig. 3(b)-2(e). Figure 2(b) shows that the electric field are confined at the right side of the crossed nanostructure, and the same symbols of electric charges are distributed on the same CR, it is because that the $r_{\text {2out }}$ for the right CR is so small. As the $r_{2 \text { out }}=90 \mathrm{~nm}$ for the right $\mathrm{CR}$, the electric field are confined at the left side of the crossed nanostructure. Especially, the inverse symbols of electric charges are distributed on the same CR when $r_{\text {2out }}=110 \mathrm{~nm}$ and $120 \mathrm{~nm}$. Thus, we can see that the electric field distribution is completely opposite when the right $\mathrm{CR}$ is smaller or bigger than left one. But the dual-Fano resonances can always be observed in the asymmetric case for the proposed crossed metasurface.

Figure 3. (a)Transmission spectra of the proposed crossed metasurface as a function of the radius $r_{\text {2out }}$ for the right CR with $\triangle r_{2}=r_{2 \text { out }}{ }^{-}{ }_{2 \text { in }}=25 \mathrm{~nm}, r_{1 \text { in }}=75 \mathrm{~nm}, r_{1 \text { out }}=100 \mathrm{~nm}$, and d $=60 \mathrm{~nm}$. (b) The electric field distribution Ez at the wavelength of $827.4 \mathrm{~nm}$ when $r_{2 o u t}=80 \mathrm{~nm}$. (c) The electric field distribution Ez 
at the wavelength of $955 \mathrm{~nm}$ when $r_{2 \text { out }}=90 \mathrm{~nm}$. (d) The electric field distribution Ez at the wavelength of $1023 \mathrm{~nm}$ when $r_{2 \text { out }}=110 \mathrm{~nm}$. (e) The electric field distribution Ez at the wavelength of $1045 \mathrm{~nm}$ when $r_{\text {2out }}=120 \mathrm{~nm}$.

The distance $\mathrm{d}$ between the center of the two CRs in $\mathrm{x}$ direction is one of important factors for regulating dual-Fano resonances in our proposed crossed ring-shaped metasurface. Thus, we investigate the transmission spectra as a function of the distance $d$ when $r_{1 \text { in }}=75 \mathrm{~nm}, r_{1 \text { out }}=100 \mathrm{~nm}, r_{2 \text { in }}=65 \mathrm{~nm}, r_{2 \text { out }}=$ $90 \mathrm{~nm}$ and the polarization of the incident light is the $x$ direction. We can see that the transmission dip 1 and dip 2 show blue shift as d increases from $60 \mathrm{~nm}$ to $120 \mathrm{~nm}$ as shown Fig. 4(a). However, the transmission dip 3 shows red shift as $d$ increases. The most interesting thing is that the transmission dip 2 gradually disappeared and the transmittance for the dip 1 increases with the increasing of the distance d. This is because the additional LSPR mode disappears as the distance $d$ increases. Then we discuss the transmission spectra as a function of $\theta$ between the direction of polarization and the $x$-axis in the $x-y$ plane when $r_{1 \text { in }}=75 \mathrm{~nm}, r_{1 \text { out }}=100 \mathrm{~nm}, r_{2 \text { in }}=65 \mathrm{~nm}, r_{2 \text { out }}=90 \mathrm{~nm}$, and $d=60 \mathrm{~nm}$. We can see that the dual-Fano resonances turn into single Fano resonance when the $\theta$ increases from 30Пto 90区, and the transmission spectra show blue shift with the increasing of $\theta$ as shown in Fig. 4(b). These results will provide guidance for the regulation of Fano resonance.

Figure 4. (a)Transmission spectra of the proposed crossed metasurface as a function of the distance $d$ with $r_{1 \text { in }}=75 \mathrm{~nm}, r_{1 \text { out }}=100 \mathrm{~nm}, r_{2 \text { in }}=65 \mathrm{~nm}, r_{2 \text { out }}=90 \mathrm{~nm}, \theta=0$. (b) Transmission spectra of the proposed crossed metasurface as a function of $\theta$ with $r_{1 \text { in }}=75 \mathrm{~nm}, r_{1 \text { out }}=100 \mathrm{~nm}, r_{2 \text { in }}=65 \mathrm{~nm}, r_{2 \text { out }}=$ $90 \mathrm{~nm}, \mathrm{~d}=60 \mathrm{~nm}$.

As is well-known, the sharp spectral lines for Fano resonance can effectively enhance the sensitivity of refractive sensing. Thus, we study sensing properties versus refractive index (RI) of external environment in the proposed crossed ring-shaped metasurface when $\theta=0 \bigotimes, r_{1 \text { in }}=75 \mathrm{~nm}, r_{1 \text { out }}=100 \mathrm{~nm}, r_{2 \text { in }}=65 \mathrm{~nm}$, $r_{2 \text { out }}=90 \mathrm{~nm}$ and $d=60 \mathrm{~nm}$. We can see that the dual-Fano resonances show red shift when RI increases from 1.00 to 1.04 as shown in Fig. 5(a). We can see that the resonant wavelength (FW) for Fano resonance peak 1 shows a linear increase with the increasing of RI as shown in Fig. 5(b). In order to investigate the sensing performance of the proposed crossed metasurface, we introduce the definition of the sensitivity as $s=\Delta \lambda / \triangle R$, where $\lambda$ is the FW for Fano resonance peak. Based on the definition of the sensitivity s, we can see that the maximum of the sensitivity Max[s] $=1010 \mathrm{~nm} / \mathrm{RIU}$ for the Fano resonance peak 1 in the proposed crossed ring-shaped metasurface. The FW of Fano resonance peak 2 versus $\mathrm{RI}$ as depicted in Fig. 5(c). We can see that FW of Fano resonance peak 2 increases as RI increases. Then the maximum of sensitivity at Fano resonance peak 2 Max[s] $=1300 \mathrm{~nm} / \mathrm{RIU}$ can be observed. The result will have great significance to design ultra-high sensitive nanosensors.

Figure 5. (a) Transmission spectrum of the proposed crossed metasurface as a function of RI with $r_{1 \text { in }}=$ $75 \mathrm{~nm}, \mathrm{r}_{1 \text { out }}=100 \mathrm{~nm}, \mathrm{r}_{2 \text { in }}=65 \mathrm{~nm}, \mathrm{r}_{2 \text { out }}=90 \mathrm{~nm}, \mathrm{~d}=60 \mathrm{~nm}, \theta=0 \mathrm{X}$. (b) The FW of Fano resonance peak 1 versus RI (c) The FWof Fano resonance peak 2 versus RI. 


\section{Conclusions}

In summary, we have studied dual-Fano resonances and high-sensitivity sensing performance in the proposed crossed ring-shaped metasurface by use of FDTD simulation method. We can find that the dual-Fano resonances in the proposed crossed ring-shaped metasurface is caused by the destructive interference among three LSPRs (LSPR at the surface of left CR, right CR, and the crossed part at the middle of the left and right CRs). In addition, we can also see that the spectra of dual-Fano resonances can be effectively tuned by the radius of the $C R$, the distance between the center of the two CRs in $x$ direction, and the polarization of the incident light. Especially, We can see that the dual-Fano resonances turn into single Fano resonance when the $\theta=90 \otimes$ or the distance $d=120 \mathrm{~nm}$ or $r_{2 \text { out }}=100 \mathrm{~nm}$. What is more, the maximum of sensitivities can reach up to $1010 \mathrm{~nm} / \mathrm{RIU}$ and $1300 \mathrm{~nm} / \mathrm{RIU}$ at Fano resonance peak 1 and Fano resonance peak 2 in our proposed crossed ring-shaped metasurface. The research results may play an important role for designing high sensitive nanosensors.

\section{Declarations}

Acknowledgments: This work was funded in part by the National Natural Science Foundation of China (NSFC) Grant No. 11847026 and 11947100, the China Postdoctoral Science Foundation Funded Project Grant No. 2019M653722, the Natural Science Basic Research Plan in Shaanxi Province of China Grant No. 2019JQ-357, the Shaanxi Key Laboratory of Optical Information Technology Foundation, the Young People's Referral Program of Shaanxi Science and Technology Association Grant No. 20170113, the Education department Program of Shaanxi Grant No. 19JK0971, the General Program of Yan'an University Grant YDY2019-20, YDQ2019-17 and YDBK2017-29.

Conflicts of Interest: The authors declare no conflicts of interest.

\section{References}

1.

Guzzinati G, Béché A, Lourenco-Martins $\mathrm{H}$ et al (2017) Probing the symmetry of the potential of localized surface plasmon resonances with phase-shaped electron beams. Nat Commun 8:14999

2.

Klar T, Perner M, Grosse S et al (1998) Surface-plasmon resonances in single metallic nanoparticles. Phys Rev Lett 80(19):4249

3.

Xu H, Li H, He Z et al (2018) Theoretical analysis of optical properties and sensing in a dual-layer asymmetric metamaterial. Opt Commun 407:250-254

4.

Cao G, Li H, Deng Y et al (2014) Plasmon-induced transparency in a single multimode stub resonator. Opt Express 22(21):25215-25223

5. 
$\mathrm{Xu} \mathrm{H}, \mathrm{Li} \mathrm{H}, \mathrm{He}$ Z et al (2017) Dual tunable plasmon-induced transparency based on silicon-air grating coupled graphene structure in terahertz metamaterial. Opt Express 25(17):20780-20790

6.

Zhang S, Genov DA, Wang Y et al (2008) Plasmon-induced transparency in metamaterials. Phys Rev Lett 101(4):047401

7.

Liu N, Langguth L, Weiss T et al (2009) Plasmonic analogue of electromagnetically induced transparency at the Drude damping limit. Nat Mater 8(9):758-762

8.

Gu J, Singh R, Liu X et al (2012) Active control of electromagnetically induced transparency analogue in terahertz metamaterials. Nat Commun 3:1151

9.

Safavi-Naeini AH, Alegre TPM, Chan J et al (2011) Electromagnetically induced transparency and slow light with optomechanics. Nature 472(7341):69-73

10.

He Z, Li H, Zhan S et al (2014) Combined theoretical analysis for plasmon-induced transparency in waveguide systems. Opt Lett 39(19):5543-5546

11.

Liu C, Li H, Xu H et al (2019) Tunable plasmon-induced transparency absorbers based on few-layer black phosphorus ribbon metamaterials. Journal of the Optical Society of America B 36(11):3060-3065 12.

Liu C, Li H, Xu H et al (2019) Slow light effect based on tunable plasmon-induced transparency of monolayer black phosphorus. J Phys D: Appl Phys 52(40):405203

13.

Chen Z, Li H, Zhan S et al (2015) Sensing characteristics based on Fano resonance in rectangular ring waveguide. Opt Commun 356:373-377

14.

Mun SE, Yun H, Choi C et al (2018) Enhancement and switching of Fano resonance in metamaterial. Advanced Optical Materials 6(17):1800545

15.

Shafiei F, Monticone F, Le KQ et al (2013) A subwavelength plasmonic metamolecule exhibiting magneticbased optical Fano resonance. Nat Nanotechnol 8(2):95

16.

Rahmani M, Luk'yanchuk B, Hong M (2013) Fano resonance in novel plasmonic nanostructures. Laser Photonics Rev 7(3):329-349

17.

Chen Z, Li P, Zhang S et al (2019) Enhanced extraordinary optical transmission and refractive-index sensing sensitivity in tapered plasmonic nanohole arrays. Nanotechnology 30(33):335201 18. 
Ebbesen TW, Lezec HJ, Ghaemi HF et al (1998) Extraordinary optical transmission through subwavelength hole arrays. Nature 391(6668):667-669

19.

Pu MB, Wang CT, Wang YQ et al (2017) Subwavelength electromagnetics below the diffraction limit. Acta Physica Sinica 66(14):144101

20.

Ma RM, Oulton RF, Sorger VJ et al (2011) Room-temperature sub-diffraction-limited plasmon laser by total internal reflection. Nat Mater 10(2):110-113

21.

Wang H, Kundu J, Halas NJ (2007) Plasmonic nanoshell arrays combine surface-enhanced vibrational spectroscopies on a single substrate. Angew Chem Int Ed 46(47):9040-9044

22.

Wang T, Dong Z, Koay EHH et al (2019) Surface-Enhanced Infrared Absorption Spectroscopy Using Charge Transfer Plasmons. ACS Photonics 6(5):1272-1278

23.

Sriram M, Zong K, Vivekchand SRC et al (2015) Single nanoparticle plasmonic sensors. Sensors 15(10):25774-25792

24.

Caucheteur C, Guo T, Albert J (2015) Review of plasmonic fiber optic biochemical sensors: improving the limit of detection. Anal Bioanal Chem 407(14):3883-3897

25.

Hsiao VKS, Zheng YB, Juluri BK et al (2008) Light-driven plasmonic switches based on au nanodisk arrays and photoresponsive liquid crystals. Adv Mater 20(18):3528-3532

26.

Lin Y, Zhang X (2017) Ultrafast Multipolar Plasmon for Unidirectional Optical Switching in a HemisphereNanoshell Array. Advanced Optical Materials 5(13):1601088

27.

Çalışkan D, Bütün B, Özcan Ş et al (2014) Spectral response modification of $\mathrm{TiO}_{2} \mathrm{MSM}$ photodetector with an LSPR filter. Opt Express 22(12):14096-14100

28.

Gao X, Zhou L, Liao Z et al (2014) An ultra-wideband surface plasmonic filter in microwave frequency. Appl Phys Lett 104(19):191603

29.

Wu C, Neuner B III, John J et al (2012) Metamaterial-based integrated plasmonic absorber/emitter for solar thermo-photovoltaic systems. J Opt 14(2):024005

30.

Aydin K, Ferry VE, Briggs RM et al (2011) Broadband polarization-independent resonant light absorption using ultrathin plasmonic super absorbers. Nat Commun 2(1):517

31. 
Li J, Chen X, Yi Z et al (2020) Broadband solar energy absorber based on monolayer molybdenum disulfide using tungsten elliptical arrays. Materials Today Energy 16:100390

32.

Li J, Chen Z, Yang H et al (2020) Tunable Broadband Solar Energy Absorber Based on Monolayer Transition Metal Dichalcogenides Materials Using Au Nanocubes. Nanomaterials 10(2):257

33.

Wang Y, Chen Z, Xu D et al (2020) Triple-band perfect metamaterial absorber with good operating angle polarization tolerance based on split ring arrays, Results in Physics, 102951

34.

Qin F, Chen Z, Chen X et al (2020) A Tunable Triple-Band Near-Infrared Metamaterial Absorber Based on Au Nano-Cuboids Array. Nanomaterials 10(2):207

35.

Cen C, Chen Z, Xu D et al (2020) High Quality Factor, High Sensitivity Metamaterial Graphene-Perfect Absorber Based on Critical Coupling Theory and Impedance Matching. Nanomaterials 10(1):95 36.

Fan S, Suh W, Joannopoulos JD (2003) Temporal coupled-mode theory for the Fano resonance in optical resonators. Journal of the Optical Society of America A 20(3):569-572

37.

Johnson AC, Marcus CM, Hanson MP et al (2004) Coulomb-modified Fano resonance in a one-lead quantum dot. Phys Rev Lett 93(10):106803

38.

Ye J, Wen F, Sobhani H et al (2012) Plasmonic nanoclusters: near field properties of the Fano resonance interrogated with SERS. Nano Lett 12(3):1660-1667

39.

Hao F, Sonnefraud Y, Dorpe PV et al (2008) Symmetry breaking in plasmonic nanocavities: subradiant LSPR sensing and a tunable Fano resonance. Nano Lett 8(11):3983-3988 40.

Wu C, Khanikaev AB, Shvets G (2011) Broadband slow light metamaterial based on a double-continuum Fano resonance. Phys Rev Lett 106(10):107403

41.

Zhan S, Peng Y, He Z et al (2016) Tunable nanoplasmonic sensor based on the asymmetric degree of Fano resonance in MDM waveguide. Sci Rep 6:22428

42.

Lu H, Liu X, Mao D et al (2012) Plasmonic nanosensor based on Fano resonance in waveguide-coupled resonators. Opt Lett 37(18):3780-3782

43.

Chen J, Yuan J, Zhang Q et al (2018) Dielectric waveguide-enhanced localized surface plasmon resonance refractive index sensing. Optical Materials Express 8(2):342-347

44. 
He Y, Zhou H, Jin Y et al (2011) Plasmon induced transparency in a dielectric waveguide. Appl Phys Lett 99(4):043113

45.

Campanella CE, De Leonardis F, Mastronardi L et al (2015) Investigation of refractive index sensing based on Fano resonance in fiber Bragg grating ring resonators. Opt Express 23(11):14301-14313

46.

Shcherbakov MR, Dobynde MI, Dolgova TV et al (2010) Full Poincaré sphere coverage with plasmonic nanoslit metamaterials at Fano resonance. Phys Rev B 82(19):193402

47.

Xu H, Zhao M, Zheng M et al (2018) Dual plasmon-induced transparency and slow light effect in monolayer graphene structure with rectangular defects. J Phys D: Appl Phys 52(2):025104 48.

Chen Z, Chen J, Wu Z et al (2014) Tunable Fano resonance in hybrid graphene-metal gratings. Appl Phys Lett 104(16):161114

49.

Zhang S, Li GC, Chen Y et al (2016) Pronounced Fano resonance in single gold split nanodisks with 15 $\mathrm{nm}$ split gaps for intensive second harmonic generation. ACS Nano 10(12):11105-11114 50 .

Fang Z, Cai J, Yan Z et al (2011) Removing a wedge from a metallic nanodisk reveals a Fano resonance. Nano Lett 11(10):4475-4479

51.

Qin F, Lai Y, Yang J et al (2017) Deep Fano resonance with strong polarization dependence in gold nanoplate-nanosphere heterodimers. Nanoscale 9(35):13222-13234

52.

Brown LV, Sobhani H, Lassiter JB et al (2010) Heterodimers: plasmonic properties of mismatched nanoparticle pairs. ACS Nano 4(2):819-832

53.

Luk'yanchuk B, Zheludev NI, Maier SA et al (2010) The Fano resonance in plasmonic nanostructures and metamaterials. Nat Mater 9(9):707-715

54.

Singh R, Cao W, Al-Naib I et al (2014) Ultrasensitive terahertz sensing with high-Q Fano resonances in metasurfaces. Appl Phys Lett 105(17):171101

55 .

Zhan S, Li H, He Z et al (2015) Sensing analysis based on plasmon induced transparency in nanocavitycoupled waveguide. Opt Express 23(16):20313-20320

56.

Tang W, Wang L, Chen X et al (2016) Dynamic metamaterial based on the graphene split ring high-Q Fano-resonnator for sensing applications. Nanoscale 8(33):15196-15204

57. 
Liu H, Zheng L, Ma P et al (2019) Metasurface generated polarization insensitive Fano resonance for high-performance refractive index sensing. Opt Express 27(9):13252-13262

58 .

Zhan S, Li H, Cao G et al (2014) Theoretical analysis and applications on nano-block loaded rectangular ring. Journal of the Optical Society of America A 31(10):2263-2267

59.

Zhan S, Li H, Cao G et al (2014) Slow light based on plasmon-induced transparency in dual-ring resonator-coupled MDM waveguide system. J Phys D: Appl Phys 47(20):205101 60.

Li B, Li H, Zeng L et al (2016) Theoretical analysis and applications in inverse T-shape structure. Journal of the Optical Society of America A 33(5):811-816

61.

He Z, Zhao J, Lu H (2020) Tunable nonreciprocal reflection and its stability in a non-PT-symmetric plasmonic resonators coupled waveguide systems. Appl Phys Express 13(1):012009

62.

He Z, Peng Y, Li B et al (2016) Aspect ratio control and sensing applications for a slot waveguide with a multimode stub. Appl Phys Express 9(7):072002

63.

Chen Z, Li H, Li B et al (2016) Tunable ultra-wide band-stop filter based on single-stub plasmonicwaveguide system. Appl Phys Express 9(10):102002

64.

Xiong C, Li H, Xu H et al (2019) Coupling effects in single-mode and multimode resonator-coupled system. Opt Express 27(13):17718-17728

65 .

He Z, Li H, Li B et al (2016) Theoretical analysis of ultrahigh figure of merit sensing in plasmonic waveguides with a multimode stub. Opt Lett 41(22):5206-5209

\section{Figures}
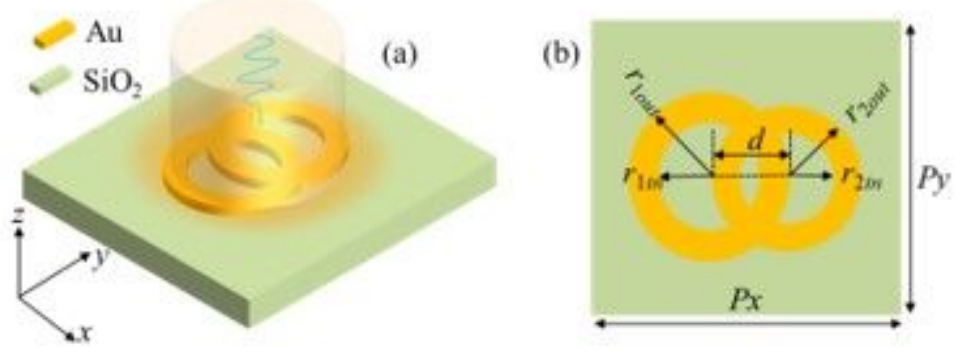

\section{Figure 1}


(a) Schematic diagram of the crossed ring-shaped metasurface, (b) $x-y$ plane view of the proposed crossed ring-shaped metasurface.

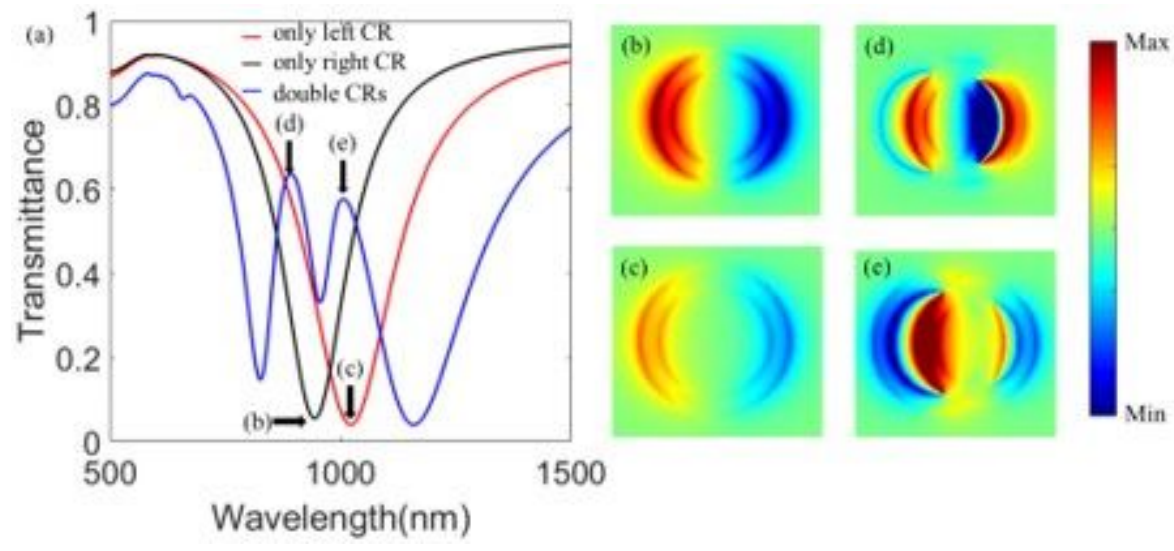

Figure 2

(a)Transmission spectra of the proposed crossed metasurface, the red line shows the transmission spectra when there is only left CR with $\mathrm{r} 1 \mathrm{in}=75 \mathrm{~nm}$ and $\mathrm{r} 1$ out=100 nm, the black line shows the transmission spectra when there is only right CR with $\mathrm{r} 2 \mathrm{in}=65 \mathrm{~nm}$ and $\mathrm{r} 2 \mathrm{out}=90 \mathrm{~nm}$, and the blue line shows the transmission spectra when there are right and left CRs arranged on the substrate with $\mathrm{r} 1 \mathrm{in}=75$ $\mathrm{nm}$, r1 out $=100 \mathrm{~nm}, \mathrm{r} 2 \mathrm{in}=65 \mathrm{~nm}, \mathrm{r} 2$ out $=90 \mathrm{~nm}$ and $\mathrm{d}=60 \mathrm{~nm}$. (b) The electric field distribution Ez at the wavelength of $994.2 \mathrm{~nm}$ in the case of only right CR. (c) The electric field distribution Ez at the wavelength of $1023 \mathrm{~nm}$ in the case of only left CR. (d) and (e) The electric field distribution Ez at the wavelength of $891.1 \mathrm{~nm}$ and $1004 \mathrm{~nm}$ in the case of double CRs.
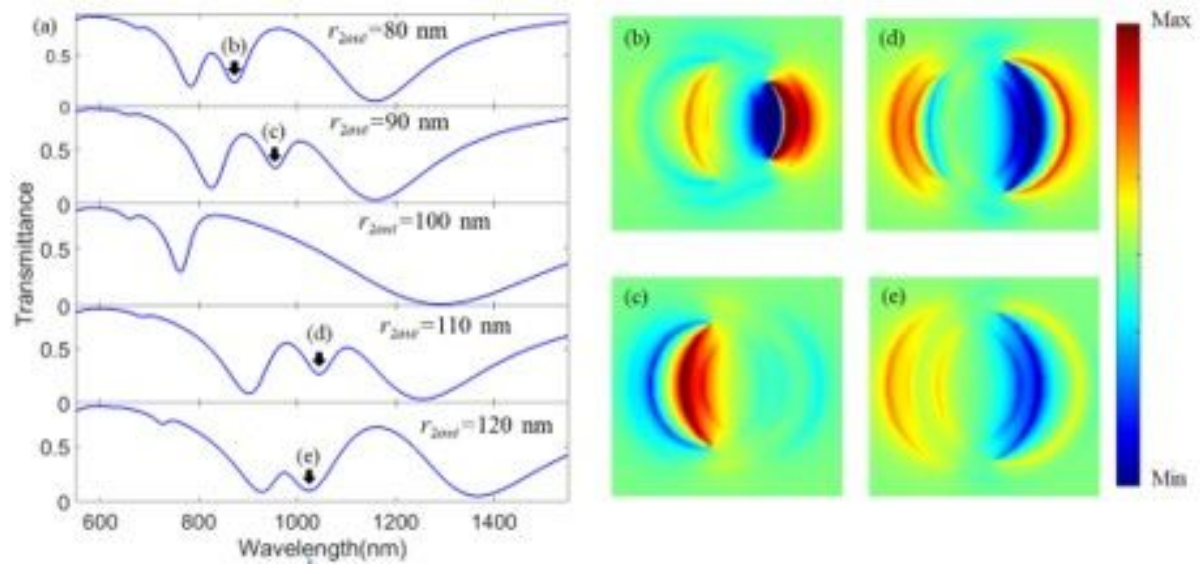

Figure 3

(a)Transmission spectra of the proposed crossed metasurface as a function of the radius r2out for the right CR with $\triangle \mathrm{r} 2=\mathrm{r} 2$ out-r2in=25 nm, r1 in=75 nm, r1 out=100 nm, and d=60 nm. (b) The electric field distribution Ez at the wavelength of $827.4 \mathrm{~nm}$ when r2out=80 nm. (c) The electric field distribution Ez at the wavelength of $955 \mathrm{~nm}$ when r2out $=90 \mathrm{~nm}$. (d) The electric field distribution Ez at the wavelength of $1023 \mathrm{~nm}$ when r2out=110 nm. (e) The electric field distribution Ez at the wavelength of $1045 \mathrm{~nm}$ when rout $=120 \mathrm{~nm}$. 

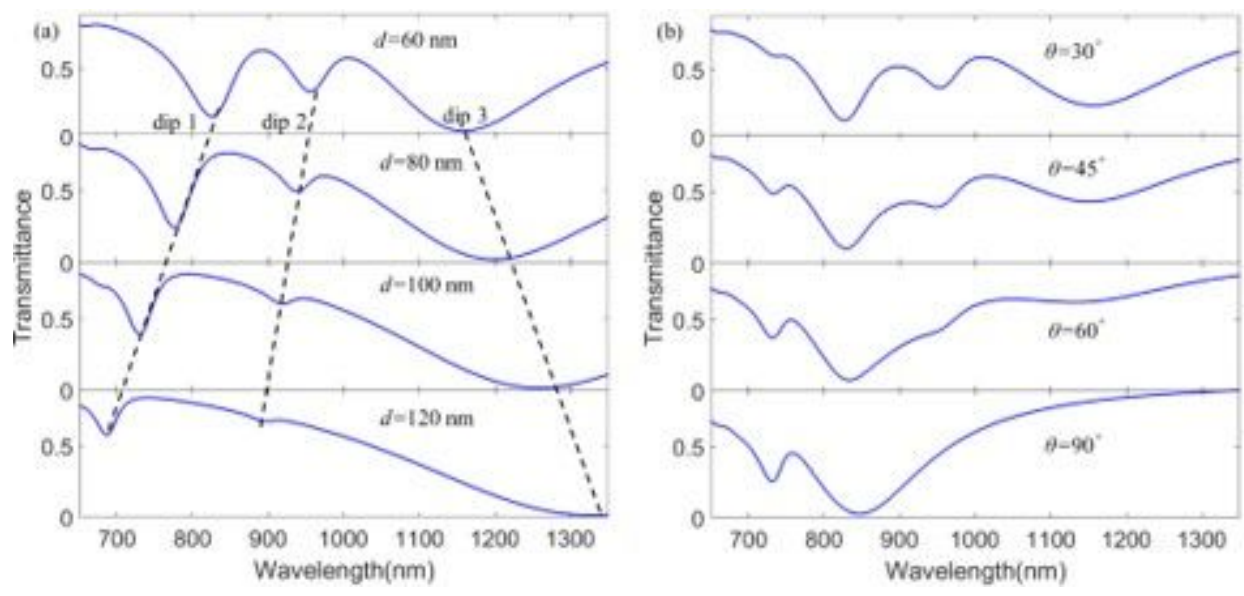

\section{Figure 4}

(a)Transmission spectra of the proposed crossed metasurface as a function of the distance $d$ with r1 in $=75 \mathrm{~nm}, \mathrm{r} 1$ out $=100 \mathrm{~nm}, \mathrm{r} 2 \mathrm{in}=65 \mathrm{~nm}, \mathrm{r} 2$ out $=90 \mathrm{~nm}, \theta=0 \bigotimes$. (b) Transmission spectra of the proposed crossed metasurface as a function of $\theta$ with $r 1$ in $=75 \mathrm{~nm}, \mathrm{r} 1$ out $=100 \mathrm{~nm}, \mathrm{r} 2 \mathrm{in}=65 \mathrm{~nm}, \mathrm{r} 2$ out $=90 \mathrm{~nm}, \mathrm{~d}=60$ $\mathrm{nm}$.
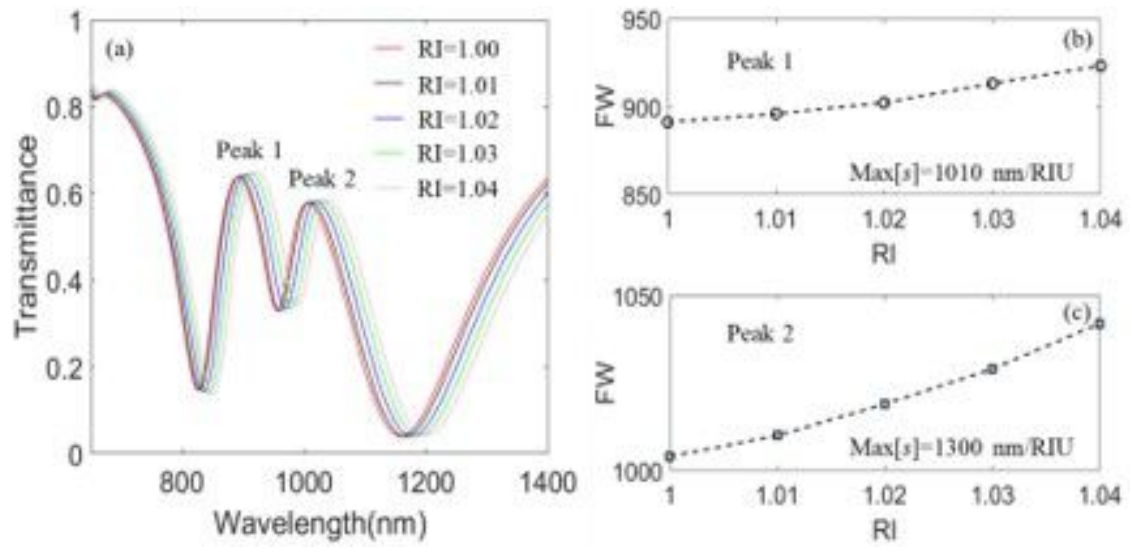

\section{Figure 5}

(a) Transmission spectrum of the proposed crossed metasurface as a function of RI with $\mathrm{r} 1 \mathrm{in}=75 \mathrm{~nm}$, r1 out $=100 \mathrm{~nm}, \mathrm{r} 2 \mathrm{in}=65 \mathrm{~nm}, \mathrm{r} 2$ out $=90 \mathrm{~nm}, \mathrm{~d}=60 \mathrm{~nm}, \theta=0 \mathrm{X}$. (b) The FW of Fano resonance peak 1 versus RI (c) The FWof Fano resonance peak 2 versus RI. 